\title{
A REAL-ANALYTIC QUASICONFORMAL EXTENSION OF A QUASISYMMETRIC FUNCTION
}

\author{
MATTI LEHTINEN
}

An increasing homeomorphism $\varphi: \mathbf{R} \rightarrow \mathbf{R}$ is $\varrho$-quasisymmetric if

$$
\varrho^{-1} \leqq(\varphi(x+t)-\varphi(x)) /(\varphi(x)-\varphi(x-t)) \leqq \varrho
$$

for all real $x$ and $t, t \neq 0$. It is well-known that every $\varrho$-quasisymmetric function can be extended to a $K$-quasiconformal mapping $f: H \rightarrow H$, where $H$ denotes the upper half-plane. This was first shown by Beurling and Ahlfors [2] who gave an explicit construction for an extension $f$. This extension is in the class $C^{1}(H)$. In the present note we shall introduce an analytic kernel into the integerals which define $f$ and thus obtain a real-analytic solution of the boundary value problem. The construction follows closely the one in [2]. Special attention, however, is paid to the estimation of the maximal dilatation $K$. The elementary but laborious computations in [2] leading to the estimate $K<\varrho^{2}$ for $\varrho>1$ seem to contain some slips. In fact, the same computations yield a somewhat sharper bound.

Remark. Recently Lehto [4] has demonstrated the existence of a real-analytic solution of the boundary value problem for quasiconformal mappings by a different, less explicit method. The maximal dilatation of his solution seems to be essentially larger than that of ours.

We state our result as

Theorem. Every $Q$-quasisymmetric function has a $K$-quasiconformal realanalytic extension to the upper half-plane. There is a number $\varrho_{0}(=1.925057 \ldots)$ such that $K<\varrho^{3 / 2}$ if $1<\varrho<\varrho_{0}$ and $K<3 \varrho^{2} / 4$ if $\varrho \geqq \varrho_{0}$.

Proof. Let $\varrho>1$ and $\varphi$ be a fixed $\varrho$-quasisymmetric function. (Since a 1quasisymmetric function is linear, there is nothing to prove in the case $\varrho=1$.) Our construction is based on the functions $u_{k}: H \rightarrow \mathbf{R}$ defined by

$$
u_{k}(x+i y)=\int_{-\infty}^{\infty} x_{k}(t) \varphi(x+y t) d t, \quad k=1,2, \ldots,
$$


where

$$
\chi_{k}(z)=c_{k} \exp \left(-(2 z)^{4^{k}}\right),
$$

and the constant $c_{k}$ is so chosen that the integral of $\varkappa_{k}$ over the real line equals $1 / 2$. The convergence of the integral in (2) for any $x+i y \in H$ is guaranteed by the inequalities [3, p. 245]

$$
\begin{aligned}
& \psi(t) \leqq 2 t^{B} \quad \text { for } \quad 0 \leqq t \leqq 1, \\
& \psi(t) \leqq(2 t)^{A} \text { for } 1 \leqq t,
\end{aligned}
$$

with $A=\log _{2}(1+\varrho), \quad B=\log _{2}\left(1+\varrho^{-1}\right), \quad$ holding for all $\varrho$-quasisymmetric $\psi$ normalized by $\psi(0)=0, \psi(1)=1$. - For future use we note that (3) applied to $t \mapsto 1-\psi(1-t)$ implies

$$
\psi\left(1-2^{-k}\right)-\psi\left(2^{-k}\right) \geqq 1-4 \varrho^{k} /(\varrho+1)^{k}, \quad k=1,2, \ldots .
$$

Lemma 1. The functions $u_{k}$ are real-analytic in $H$ and

for every real $x_{0}$.

$$
\lim _{z \rightarrow x_{0}} u_{k}(z)=\varphi\left(x_{0}\right) / 2
$$

Proof. Fix $x_{0}+i y_{0}$ in $H$. Inequality (4) implies the uniform and absolute convergence of the integrals

$$
\begin{gathered}
g_{k}(z, w)=\int_{-\infty}^{\infty} \chi_{k}((t-z) / w) \varphi(t) d t \\
\int_{-\infty}^{\infty} \chi_{k}^{\prime}((t-z) / w) \varphi(t) d t, \quad \int_{-\infty}^{\infty} t \chi_{k}^{\prime}((t-z) / w) \varphi(t) d t
\end{gathered}
$$

in a sufficiently small polydisc $U \subset \mathbf{C} \times \mathbf{C}$, with center $\left(x_{0}, y_{0}\right)$. It follows by a standard argument that $g_{k}$ has complex derivatives with respect to $z$ and $w$ in $U$. Hence $g_{k}$ is holomorphic at $\left(x_{0}, y_{0}\right)$; since $u_{k}(x+i y)=y g_{k}(x, y)$ for $x+i y \in H$, the function $u_{k}$ is real-analytic at $x_{0}+i y_{0}$. To prove (6), let $x_{n}+i y_{n}$ with $y_{n}>0$ converge to $x_{0}$ and apply Lebesgue's convergence theorem to the integrals

$$
\int_{-\infty}^{\infty} x_{k}(t)\left(\varphi\left(x_{n}+y_{n} t\right)-\varphi\left(x_{0}\right)\right) d t .
$$

By (4) they are majorized by an integrable function and thus converge to zero as $n$ tends to infinity.

The following two lemmas contain the necessary estimates of the partial derivatives of $u_{k}$.

\section{Lemma 2.}

uniformly in $H$.

$$
\limsup _{k \rightarrow \infty} \frac{\left|\left(u_{k}\right)_{y}(z)\right|}{\left(u_{k}\right)_{x}(z)} \leqq \frac{\varrho-1}{2(\varrho+1)}
$$


Proof. Given $z=x+i y \in H$, set

$$
\psi(t)=\varphi(x+y t), \quad t_{k}=\left(2 \cdot 4^{k 4^{-k}}\right)^{-1}, \quad \text { and } \quad a_{k}=\psi\left(t_{k}\right)-\psi(0)
$$

Observe that $t \mapsto t \varkappa_{k}(t)$ takes its largest value at $t_{k}$. Differentiation of (2) and a subsequent integration by parts shows that

$$
y\left(u_{k}\right)_{x}(z)=\int_{-\infty}^{\infty} x_{k}(t) d \psi(t), \quad y\left(u_{k}\right)_{y}(z)=\int_{-\infty}^{\infty} t x_{k}(t) d \psi(t) .
$$

Because $\psi$ is increasing and $\varkappa_{k}$ is positive, and because of (1),

$$
\int_{-\infty}^{\infty} \varkappa_{k}(t) d \psi(t)>\int_{-t_{k}}^{t_{k}} \varkappa_{k}(t) d \psi(t)>\left(1+\varrho^{-1}\right) a_{k} \varkappa_{k}\left(t_{k}\right) .
$$

On the other hand,

$$
\int_{-\infty}^{t_{k}} t \chi_{k}(t) d \psi(t)<\int_{-\infty}^{\infty} t \chi_{k}(t) d \psi(t)<\int_{-t_{k}}^{\infty} t \varkappa_{k}(t) d \psi(t) .
$$

The integral on the right hand side of (7) is smaller than

$$
\begin{gathered}
\varkappa_{k}\left(t_{k}\right) \int_{-t_{k}}^{0} t d \psi(t)+\varkappa_{k}(0) \int_{0}^{t_{k}} t d \psi(t)+\varkappa_{k}\left(t_{k}\right)\left(\psi\left(1-t_{k}\right)-\psi\left(t_{k}\right)\right) \\
+x_{k}\left(1-t_{k}\right) \varrho a_{k}+a_{k} t_{k} \sum_{n=2}^{\infty} n \varkappa_{k}\left(n t_{k}\right) \varrho^{n} .
\end{gathered}
$$

By a lemma of Beurling and Ahlfors [1, p. 67]

and similarly

$$
\begin{gathered}
\int_{-t_{k}}^{0} t d \psi(t)=t_{k} \psi\left(-t_{k}\right)-\int_{-t_{k}}^{0} \psi(t) d t \\
\leqq-\frac{t_{k}}{\varrho+1}\left(\psi(0)-\psi\left(-t_{k}\right)\right) \leqq \frac{-t_{k} a_{k}}{\varrho(\varrho+1)},
\end{gathered}
$$

By (3),

$$
\int_{0}^{t_{k}} t d \psi(t) \leqq \frac{t_{k} a_{k} \varrho}{\varrho+1}
$$

$$
\psi\left(1-t_{k}\right)-\psi\left(t_{k}\right)<2\left(\psi\left(2 t_{k}\right)-\psi\left(t_{k}\right)\right)\left(1-2 t_{k}\right)^{B} \leqq 2 a_{k} \varrho\left(1-2 t_{k}\right)^{B} .
$$

The left hand side of (7) can be estimated from below in the same way. Combining the estimates, dividing by $a_{k}$, and observing that $t_{k}, x_{k}(0)$ and $x_{k}\left(t_{k}\right)$ tend to $1 / 2$ while $x_{k}\left(1-t_{k}\right)$ and the infinite sum in (8) tend to zero as $k \rightarrow \infty$, we obtain the desired inequality. 
Lemma 3. If $\lambda=1 / 2$ or $-1 / 2$,

uniformly for $x+i y \in H$.

$$
\limsup _{k \rightarrow \infty} \frac{\left(u_{k}\right)_{x}(x+\lambda y+i y)}{\left(u_{k}\right)_{x}(x-\lambda y+i y)} \leqq \varrho,
$$

Proof. We may suppose $\lambda=1 / 2$. Then, with $\psi$ as in the previous lemma and by (5) and (1),

$$
\begin{gathered}
y\left(u_{k}\right)_{x}(x-y / 2+i y)>\chi_{k}\left(2^{-1}-2^{-k}\right)\left(\psi\left(-2^{-k}\right)-\psi\left(-1+2^{-k}\right)\right) \\
\geqq \varrho^{-1} \varkappa_{k}\left(2^{-1}-2^{-k}\right)\left(1-4 \varrho^{k} /(\varrho+1)^{k}\right)(\psi(1)-\psi(0)) .
\end{gathered}
$$

In the other direction we have

$$
\begin{aligned}
& y\left(u_{k}\right)_{x}(x+y / 2+i y)<x_{k}(0)(\psi(1)-\psi(0)) \\
+ & x_{k}\left(2^{-1}\right)\left(\psi\left(1+2^{-k}\right)-\psi(1)+\psi(0)-\psi\left(-2^{-k}\right)\right) \\
+ & x_{k}\left(2^{-1}+2^{-k}\right)(\psi(2)-\psi(1)+\psi(0)-\psi(-1)) \\
+ & 2(\psi(1)-\psi(0)) \sum_{n=1}^{\infty} x_{k}\left(2^{-1}+n\right) \varrho^{n+1} .
\end{aligned}
$$

By (3) and (1)

$$
\psi\left(1+2^{-k}\right)-\psi(1) \leqq 2 \cdot 2^{-k B} \varrho(\psi(1)-\psi(0))
$$

and

$$
\psi(0)-\psi\left(-2^{-k}\right) \leqq \varrho\left(\psi\left(2^{-k}\right)-\psi(0)\right) \leqq 2 \cdot 2^{-k B} \varrho(\psi(1)-\psi(0)) .
$$

To obtain the asserted inequality it now suffices to combine the estimates, divide by $\psi(1)-\psi(0)$ and observe that $x_{k}\left(2^{-1}+2^{-k}\right)$ and the infinite sum in (9) tend to zero as $k \rightarrow \infty$.

We now proceed to the definition of the quasiconformal extension. To this end, set $\alpha_{k}(z)=u_{k}(z+y / 2), \beta_{k}(z)=u_{k}(z-y / 2)(z=x+i y)$ and

$$
f_{k}=\alpha_{k}+\beta_{k}+\operatorname{ir}\left(\alpha_{k}-\beta_{k}\right),
$$

where $r$ is a positive parameter. By the chain rule, the partial derivatives of $\alpha_{k}$ and $\beta_{k}$ are

$$
\begin{aligned}
& \left(\alpha_{k}\right)_{x}(z)=\left(u_{k}\right)_{x}(z+y / 2), \quad\left(\beta_{k}\right)_{x}(z)=\left(u_{k}\right)_{x}(z-y / 2), \\
& \left(\alpha_{k}\right)_{y}(z)=\left(u_{k}\right)_{x}(z+y / 2) / 2+\left(u_{k}\right)_{y}(z+y / 2), \\
& \left(\beta_{k}\right)_{y}(z)=-\left(u_{k}\right)_{x}(z-y / 2) / 2+\left(u_{k}\right)_{y}(z-y / 2) .
\end{aligned}
$$

Lemma 4. There is a $k_{0}$ such that for $k \geqq k_{0} f_{k}: H \rightarrow H$ is a bijection.

Proof. One easily sees that $f_{k}$ is one to one if and only if the pair of equations

$$
\begin{aligned}
& \alpha_{k}(x+i y)=a \\
& \beta_{k}(x+i y)=b
\end{aligned}
$$

has one and only one solution for all $a, b$ with $a>b$. But (10) and (11) define 
for $x<\varphi^{-1}(2 a)$ resp. $x>\varphi^{-1}(2 b)$ a decreasing resp. increasing curve $y=g_{k}(x)$, $y=h_{k}(x)$. These intersect certainly if $-g_{k}^{\prime}$ and $h_{k}^{\prime}$ are bounded above. But

and

$$
-g_{k}^{\prime}(x)=\left(1 / 2+\left(u_{k}\right)_{y}(z+y / 2) /\left(u_{k}\right)_{x}(z+y / 2)\right)^{-1},
$$

$$
h_{k}^{\prime}(x)=\left(1 / 2-\left(u_{k}\right)_{y}(z-y / 2) /\left(u_{k}\right)_{x}(z-y / 2)\right)^{-1},
$$

so that the assertion follows from Lemma 2 .

We have shown that $f_{k}$ is - for $k$ large enough - a real-analytic homeomorphism with correct boundary values. Next we want to estimate the maximal dilatation of $f_{k}$. Denote the ratios $\left(\alpha_{k}\right)_{y} /\left(\alpha_{k}\right)_{x},\left(\beta_{k}\right)_{y} /\left(\beta_{k}\right)_{x},\left(\alpha_{k}\right)_{x} /\left(\beta_{k}\right)_{x}$, evaluated at $z$, by $\xi, \eta, \zeta$, respectively. If $\varrho^{\prime}>\varrho$ is arbitrary we can, by Lemmas 2 and 3 , fix a $k \geqq k_{0}$ such that $\xi$ and $\eta$ lie between $\left(1+\varrho^{\prime}\right)^{-1}$ and $\varrho^{\prime}\left(1+\varrho^{\prime}\right)^{-1}$ for all $z \in H$. We denote $f_{k}$ by $f$ and its dilatation quotient at $z$ by $D$. As in [2] we compute

$$
D+D^{-1}=\frac{1}{2 r(\xi+\eta)}\left(\left(\zeta\left(1+\xi^{2}\right)+\zeta^{-1}\left(1+\eta^{2}\right)\right)\left(1+r^{2}\right)+2(1-\xi \eta)\left(1-r^{2}\right)\right) .
$$

The right hand side of (12) is invariant under the transformation $(\xi, \eta, \zeta)$ $\mapsto\left(\eta, \xi, \zeta^{-1}\right)$. Hence it suffices to consider the case $\xi \geqq \eta$. With this condition one easily sees that for $\xi, \eta$, and $r$ fixed, the right hand side of (12) is maximized by $\zeta=\varrho^{\prime}$. It follows that

where

$$
D+D^{-1} \leqq a(\xi, \eta) r+b(\xi, \eta) r^{-1}=F(\xi, \eta, r),
$$

and

$$
a(\xi, \eta)=\frac{\left(\varrho^{\prime}-1\right)^{2}+\left(\varrho^{\prime} \xi+\eta\right)^{2}}{2 \varrho^{\prime}(\xi+\eta)}
$$

$$
b(\xi, \eta)=\frac{\left(\varrho^{\prime}+1\right)^{2}+\left(\varrho^{\prime} \xi-\eta\right)^{2}}{2 \varrho^{\prime}(\xi+\eta)} .
$$

The problem is to minimize with respect to $r$ the maximum of $F$ in the triangle $T=\left\{(\xi, \eta) \mid\left(\varrho^{\prime}+1\right)^{-1} \leqq \eta \leqq \xi \leqq \varrho^{\prime}\left(\varrho^{\prime}+1\right)^{-1}\right\}$. A routine computation shows that $F$ can attain its maximum in $T$ only at the vertices of $T$. Denote

where

$$
\begin{aligned}
F\left(\left(\varrho^{\prime}+1\right)^{-1},\left(\varrho^{\prime}+1\right)^{-1}, r\right) & =F_{1}(r)=a_{1} r+b_{1} r^{-1}, \\
F\left(\varrho^{\prime}\left(\varrho^{\prime}+1\right)^{-1},\left(\varrho^{\prime}+1\right)^{-1}, r\right) & =F_{2}(r)=a_{2} r+b_{2} r^{-1}, \\
F\left(\varrho^{\prime}\left(\varrho^{\prime}+1\right)^{-1}, \varrho^{\prime}\left(\varrho^{\prime}+1\right)^{-1}, r\right) & =F_{3}(r)=a_{3} r+b_{3} r^{-1},
\end{aligned}
$$

$$
\begin{array}{ll}
a_{1}=\frac{\varrho^{\prime 3}-\varrho^{\prime 2}+2}{4 \varrho^{\prime}}, & b_{1}=\frac{\varrho^{\prime 4}+4 \varrho^{\prime 3}+7 \varrho^{\prime 2}+2 \varrho^{\prime}+2}{4 \varrho^{\prime 2}+4 \varrho^{\prime}}, \\
a_{2}=\frac{\varrho^{\prime 4}+1}{\varrho^{\prime 3}+2 \varrho^{\prime 2}+\varrho^{\prime}}, & b_{2}=\varrho^{\prime}+\varrho^{\prime-1}, \\
a_{3}=\frac{2 \varrho^{\prime 3}-\varrho^{\prime}+1}{4 \varrho^{\prime 2}}, & b_{3}=\frac{2 \varrho^{\prime 4}+2 \varrho^{\prime 3}+7 \varrho^{\prime 2}+4 \varrho^{\prime}+1}{4 \varrho^{\prime 3}+4 \varrho^{\prime 2}} .
\end{array}
$$


Every $F_{i}$ is minimized at $r_{i}=\left(b_{i} / a_{i}\right)^{1 / 2}$ and the only poossible positive root $r_{i j}$ of the equation $F_{i}(r)=F_{j}(r), i \neq j$, satisfies

$$
r_{i j}^{2}=\left(b_{i}-b_{j}\right) /\left(a_{j}-a_{i}\right) .
$$

It is clear that $\max \left\{F_{1}, F_{2}, F_{3}\right\}$ is minimized by some $r_{i}$ or some $r_{i j}$. We observe that $b_{1}>b_{2}>b_{3}$ for all $\varrho^{\prime}>1$. We first compare $F_{1}$ and $F_{2}$. A computation shows that $r_{1}>r_{2}$ for all $\varrho^{\prime}>1$. It follows that $\max \left\{F_{1}, F_{2}\right\}$ is minimized at $r_{2}$ if $r_{12} \leqq r_{2}$, at $r_{12}$ if $r_{2}<r_{12}<r_{1}$ and at $r_{1}$ if $r_{1} \leqq r_{12}$ or $r_{12}$ is imaginary. The first alternative is found out to hold for $\varrho^{\prime} \leqq \varrho_{2}=1.387808 \ldots$, the second for $\varrho_{2}<\varrho^{\prime}<$ $<\varrho_{1}=1.969351 \ldots$, and the third for $\varrho^{\prime} \geqq \varrho_{1}$. Further calculations show that $r_{23}$ is real only for $\varrho^{\prime}<1.959413 \ldots<\varrho_{1}$. Thus if $\varrho^{\prime}>\varrho_{1}$, then $F_{1}(r)>F_{3}(r)$ for all $r$. On the other hand the inequalities $r_{2}<r_{23}$ and $r_{12}<r_{23}$ are found out to hold for all $\varrho^{\prime}>1$ for which $r_{23}$ is real. It follows, in particular, that $F_{2}\left(r_{2}\right)>F_{3}\left(r_{2}\right)$ and $F_{2}\left(r_{12}\right)>F_{3}\left(r_{12}\right)$ for $1<\varrho^{\prime}<\varrho_{1}$.

Altogether, we have shown that the maximal dilatation $K$ of $f$ satisfies the inequalities

for $\varrho_{2}<\varrho^{\prime}<\varrho_{1}$, and

$$
\begin{aligned}
& K+K^{-1} \leqq F_{2}\left(r_{2}\right)=2\left(a_{2} b_{2}\right)^{1 / 2}, \text { for } \varrho^{\prime} \leqq \varrho_{2}, \\
& K+K^{-1} \leqq F_{1}\left(r_{12}\right)=\frac{a_{2} b_{1}-a_{1} b_{2}}{\left(\left(a_{2}-a_{1}\right)\right.} \frac{\left.\left(b_{1}-b_{2}\right)\right)^{1 / 2}}{},
\end{aligned}
$$

$$
K+K^{-1} \leqq F_{1}\left(r_{1}\right)=2\left(a_{1} b_{1}\right)^{1 / 2}, \quad \text { for } \quad \varrho_{1} \leqq \varrho^{\prime} .
$$

Now if $\varrho^{\prime}<\varrho_{2}$ the inequality $K<\varrho^{\prime 3 / 2}$ holds if

$$
4 a_{2} b_{2}<\varrho^{\prime 3}+\varrho^{\prime-3}+2 .
$$

A computation shows that the inequality is indeed valid. For $\varrho^{\prime}>\varrho_{1}$ the inequality $K<c \varrho^{\prime 2}$ is satisfied if

$$
4 a_{1} b_{1}<c^{2} \varrho^{\prime 4}+c^{-2} \varrho^{\prime-4}+2
$$

If (13) is written explicitly one sees that it is satisfied by any $c>1 / 2$ for $\varrho^{\prime}$ large enough and for all $\varrho^{\prime}>\varrho_{1}$ e.g. by $c=3 / 4$. The computations are rather complicated in the case $\varrho_{2}<\varrho^{\prime}<\varrho_{1}$. It turns out that the inequality $K<\varrho^{\prime 3 / 2}$ holds for $\varrho^{\prime}<\varrho_{0}=1.925057 \ldots$ while for $\varrho_{0} \leqq \varrho^{\prime}<\varrho_{1}$ the inequality $K<c \varrho^{\prime 2}$ holds with $c=3 / 4$. Since $\varrho^{\prime}>\varrho$ was arbitrary, the proof of the theorem is completed.

Remark. The above estimation of $K$ is valid, with $\varrho^{\prime}$ replaced by $\varrho$, for the original extension of Beurling and Ahlfors. As pointed out in the proof, actually $K<c(\varrho) \varrho^{2}$ with $\lim _{\varrho \rightarrow \infty} c(\varrho)=1 / 2$. In view of the universal bound $K<8 \varrho$, due to Reed [5], this is, however, of little interest. 


\title{
References
}

[1] Ahlfors, L. V.: Lectures on quasiconformal mappings. - D. van Nostrand Company, Inc., Princeton, N. J., 1966.

[2] Beurling, A., and L. V. Ahlfors: The boundary correspondence under quasiconformal mappings. - Acta Math. 96, 1956, 125-142.

[3] Kelingos, J. A.: Boundary correspondence under quasiconformal mappings. - Michigan Math. J. 13, 1966, 235-249.

[4] LeHTo, O.: On the boundary value problem for quasiconformal mappings. - To appear.

[5] ReED, T. J.: Quasiconformal mappings with given boundary values. - Duke Math. J. 33, 1966, $459-464$.

\author{
University of Helsinki \\ Department of Mathematics \\ SF-00100 Helsinki 10 \\ Finland
}

Received 25 October 1976 